\title{
A FAMÍlIA NO CENÁRIO HOSPITALAR PEDIÁTRICO A PARTIR DA DÉCADA DE 1990: UMA REVISÃO INTEGRATIVA
}

Damariz Barros de Albuquerque ${ }^{1}$, Rita de Cássia Melão de Morais² ${ }^{2}$ Isabela Fornerolli de Macedo $^{3}$, Rosana Fidelis Coelho Vieira ${ }^{4}$,Tania Vignuda de Souza ${ }^{5}$

RESUMO: Revisão integrativa com objetivo de analisar a produção científica nas bases de dados online referente à presença do familiar na unidade de internação pediátrica. O levantamento bibliográfico abrangeu a busca nas bases de dados LILACS, MEDLINE, CINAHL e COCHRANE no período de maio a junho de 2012, sendo identificados 15 artigos científicos que compuseram a amostra do estudo. Os resultados apontaram que há preocupação dos enfermeiros em desenvolver pesquisas cujo participante seja a família da criança hospitalizada em relação aos seguintes temas: vivencias/ sentimentos da família; interação entre a equipe de enfermagem e o familiar/acompanhante; e necessidades da família durante a hospitalização da criança. Conclui-se que os estudos versam, de modo geral, sobre a busca incessante dos pesquisadores em entender os familiares, dando voz aos mesmos. Em contrapartida, a prática não reflete mudanças de comportamento da equipe de saúde no que se refere à inclusão da família como parte do cuidado.

DESCRITORES: Família; Criança hospitalizada; Enfermagem Pediátrica.

\section{THE FAMILY IN THE PEDIATRIC HOSPITAL SETTING SINCE THE 1990'S: AN INTEGRATIVE REVIEW}

\begin{abstract}
This integrative review aimed to analyze scientific production in the online databases referent to the presence of the family member in the pediatric inpatient unit. The bibliographic survey covered the search in the LILACS, MEDLINE, CINAHL and COCHRANE databases in the period May - June 2012, 15 scientific articles being identified, which made up the sample study. The results indicate that nurses are concerned with undertaking research whose participants are family of the hospitalized child, in relation to the following themes: the family's experiences/feelings; interaction between the nursing team and the family member/companion; and, the family's needs during the child's inpatient treatment. It is concluded that the studies address, in a general way, on the researchers' incessant search to understand the family members, giving a voice to the same. In contrast, the practice does not reflect changes of behavior in the nursing team regarding the inclusion of the family as part of the care. DESCRIPTORS: Family; Hospitalized child; Pediatric Nursing.

\section{LA FAMILIA EN EL ÁMBITO HOSPITALAR PEDIÁTRICO A PARTIR DE LA DÉCADA DE 1990: UNA REVISIÓN INTEGRATIVA}

RESUMEN: Revisión integrativa cuyo objetivo fue analizar la producción científica en las bases de datos online referente a la presencia del familiar en la unidad de internación pediátrica. El aporte bibliográfico abarcó la búsqueda en las bases de datos LILACS, MEDLINE, CINAHL y COCHRANE en el período de mayo a junio de 2012, siendo identificados 15 artículos científicos que formaron parte de la muestra del estudio. Los resultados apuntaron que hay preocupación de los enfermeros en desarrollar investigaciones cuyo participante sea la familia del niño hospitalizado asociándose a los siguientes temas: vivencias/sentimientos de la familia; interacción entre el equipo de enfermería y el familiar/acompañante; y necesidades de la familia durante la hospitalización del niño. Se concluye que los estudios discurren, de modo general, sobre la búsqueda incesante en entender los familiares, dando voz a los mismos. Por otro lado, la práctica no reflecte cambios de comportamiento del equipo de salud acerca de la inclusión de la familia como parte del cuidado.

DESCRIPTORES: Familia; Niño hospitalizado; Enfermería pediátrica.

${ }^{1}$ Enfermeira do Instituto Estadual de Cardiologia Aloysio de Castro e Hospital Pró-Cardíaco. Mestre em Enfermagem.

${ }^{2}$ Enfermeira. Mestre em Enfermagem. Professora do Departamento de Enfermagem Materno-Infantil da Escola de Enfermagem Anna Nery da Universidade Federal do Rio de Janeiro - EEAN UFRJ. Membro do Núcleo de Pesquisa em Enfermagem em Saúde da Criança e do Adolescente -NUPESC e do Grupo de Pesquisa Saúde da Criança: Cenário Hospitalar.

${ }^{3}$ Enfermeira. MestrandaemEnfermagempelaEEANUFRJ.ProfessoraSubstitutadoDepartamentodeEnfermagem Materno-InfantildaFaculdade deEnfermagem da Universidade do Estado do Rio de Janeiro. Membro do NUPESC e do Grupo de Pesquisa Saúde da Criança: Cenário Hospitalar.. ${ }^{4}$ Enfermeira da Unidade de Tratamento Intensivo Pediátrico do Instituto Nacional do Câncer. Mestranda em Enfermagem pela EEAN UFRJ.Membro do NUPESC e do Grupo de Pesquisa Saúde da Criança: Cenário Hospitalar.

${ }^{5}$ Enfermeira. Doutora em Enfermagem. Professora da EEAN UFRJ. Membro do NUPESC e do Grupo de Pesquisa Saúde da Criança: Cenário Hospitalar.

Autor correspondente:

Recebido: $28 / 02 / 2013$

Rita de Cássia Melão de Morais. Aprovado: 19/09/2013

Universidade Federal do Rio de Janeiro

Rua Conde de Bonfim, 897, - 20530-000 - Rio de Janeiro-RJ-Brasil

E-mail: ritamelao@gmail.com 


\section{INTRODUÇÃO}

No Brasil, o sujeito família vem sendo estudado, gradativamente, ao longo da década de 90 do Século $\mathrm{XX}$, especificamente quando se trata do cenário da hospitalização infantil. Esta afirmativa vai ao encontro da inserção de um familiar ou responsável legal para o acompanhamento da criança ou adolescente hospitalizado como garantia de um direito constituído pela Lei 8.069/90 do Estatuto da Criança e do Adolescente $(\mathrm{ECA})^{(1)}$. A promulgação desta Lei constituiu a garantia de inserção do familiar em um cenário que outrora era de domínio dos profissionais de saúde tendo como consequência a ocorrência de conflitos nas relações estabelecidas entre os familiares e equipe de enfermagem ${ }^{(2)}$.

O termo família é identificado de variadas formas dependendo do julgamento de valores ou da estrutura de referencia do indivíduo, ou seja, não existe uma definição universal, o termo família deve ser considerado como o que o indivíduo considera que ele $\operatorname{seja}^{(3)}$. E, é a partir da prática profissional em uma enfermaria pediátrica que para atender ao direito da criança hospitalizada, se verifica a presença do familiar por longos períodos durante a sua hospitalização em detrimento do acompanhamento da criança saudável que encontra-se no domicílio, de seu emprego, do cônjuge. Em contrapartida, a família não costuma ser considerada pela equipe de saúde como uma unidade de cuidado. Mais precisamente, a família é considerada como um elemento importante para o fornecimento de informações sobre a criança, além de auxiliar nos cuidados já desenvolvidos no domicílio( ${ }^{(4)}$.

A infraestrutura por parte das instituições hospitalares ainda não oferece conforto adequado para o membro da família que acompanha a criança. Resultados de um estudo desenvolvido em Goiânia - Goiás apontam que as Unidades de Internação Pediátricas, não contemplam condições adequadas para a permanência conjunta do binômio criança e acompanhante evidenciada pela falta de instalações ou inadequação da unidade para esta permanência conjunta ${ }^{(5-6)}$.

O cansaço físico da família foi evidenciado pelos sujeitos ora por não conseguirem dormir devido ao estado de saúde da criança, assim como também pelo fato das condições de acomodação não serem adequadas para a permanência conjunta, apesar da existência de poltronas para descanso ${ }^{(7)}$. Desse modo, a equipe de enfermagem pediátrica necessita estar sensibilizada e instrumentalizada para acolher e compreender a criança como um ser vulnerável, bem como seu familiar que está susceptível ao desgaste físico e emocional ${ }^{(8)}$.

Cabe destacar que antes da promulgação do ECA, não eram desenvolvidas reflexões acerca da presença/ permanência do acompanhante da criança no ambiente da unidade de internação pediátrica e as normas e padrões de construções e instalações de serviços de saúde tinham como referência a Portaria 282/82 do Ministério da Saúde. Somente 12 anos depois de instituído o direito da criança em ter um responsável que o acompanhe, surgIU a RDC n. 50/2002 que estipula condições mínimas para a permanência deste novo elemento no cenário hospitalar pediátrico ${ }^{(9)}$.

Tomando como marco o ECA, que determinou mudanças na assistência à criança hospitalizada, incluindo a família na unidade de internação pediátrica, esta pesquisa tem como questionamento: Qual a produção científica sobre a temática "familiar/acompanhante da criança hospitalizada"? Como objeto o conhecimento científico sobre a família no cenário hospitalar durante a hospitalização da criança desde a promulgação do ECA na década de 1990.

Desta forma, o objetivo do presente estudo consiste em analisar a produção científica publicada nas bases de dados online referente à presença do familiar na unidade de internação pediátrica.

\section{MÉTODO}

A fim de alcançar o objetivo proposto, optou-se pelo método da revisão integrativa, visto que ele possibilita reunir e sintetizar resultados de pesquisas sobre um determinado tema ou questão, de maneira sistemática e ordenada, contribuindo para o aprofundamento do tema investigado, além de apontar lacunas do conhecimento que precisam ser preenchidas com a realização de novas pesquisas(10). O levantamento bibliográfico foi realizado no período de maio a junho de 2012, e relativo ao período de 1990 a 2011, nas bases de dados: Medical Literature Analysis and Retrieval Sistem online (MEDLINE); Literatura Latino-Americana e do Caribe em Ciências da Saúde (LILACS), CINAHL e Biblioteca Cochrane (COCHRANE).

Foram utilizados, para a busca dos artigos, os descritores família, mãe e criança hospitalizada. Os critérios utilizados para sua seleção foram: artigos publicados em português, inglês ou espanhol; cuja autoria fosse de pesquisadores brasileiros e cujos participantes dos estudos fossem familiares de crianças hospitalizadas, em pesquisa realizada durante o período de hospitalização da criança, em cenário de Unidade de Internação Pediátrica (UIP) ou Alojamento Conjunto Pediátrico (ACP). 
Os critérios de exclusão foram: estudos de revisão, relatos de caso, reflexão ou comunicação e estudos que se repetissem entre as bases de dados, considerando-se apenas um. Apesar do ECA se tratar de uma Lei Brasileira, todos os estudos internacionais publicados dentro do recorte temporal estabelecido, e que se adequaram aos critérios de inclusão, foram considerados para análise, tendo em vista serem de autores brasileiros.

O cruzamento dos referidos descritores foi realizado da seguinte forma: família and criança hospitalizada; mãe and criança hospitalizada. Uma vez acessados os títulos e resumos das publicações, 68 artigos foram selecionados e adquiridos na íntegra. De posse de todas as cópias, procedeu-se a leitura integral e crítica dos 68 artigos quanto aos critérios de inclusão, ficando a amostra final composta por 15 artigos científicos.

Para facilitar a coleta de dados, foi desenvolvido um quadro demonstrativo, com informações relativas a cada estudo e contendo itens como: ano de publicação; título e autores, local de publicação, tipo de estudo, objetivos, resultados, referencial metodológico e teórico. A utilização do referido quadro permitiu a sucinta organização dos dados, facilitando a comparação dos estudos em tópicos específicos. Os resultados extraídos de cada estudo foram analisados de forma descritiva, discutidos à luz da literatura específica.

\section{RESULTADOS}

Constata-se que a amostra final foi identificada majoritariamente na base de dados LILACS, seguida pela base de dados MEDLINE e, por fim, CINAHL. A identificação de cada um desses artigos encontra-se descrita no quadro a seguir.

Quadro 1 - Identificação dos artigos selecionados sobre presença do familiar na unidade de internação pediátrica

\begin{tabular}{|l|l|l|l|}
\hline Base de Dados & Ano da Publicação & Título do Artigo & Estado/País \\
\hline LILACS & 2011 & $\begin{array}{l}\text { O apoio social ao familiar cuidador durante a internação } \\
\text { hospitalar da criança }\end{array}$ & RS/BR \\
\hline CINAHL & 2010 & $\begin{array}{l}\text { A família vivenciando a doença e a hospitalização da } \\
\text { criança: estudo qualitativo }\end{array}$ & SP/BR \\
\hline LILACS & 2010 & $\begin{array}{l}\text { As mulheres cuidadoras leigas acompanhantes de crianças } \\
\text { com câncer no contexto hospitalar }\end{array}$ & RS/BR \\
\hline LILACS & 2010 & $\begin{array}{l}\text { Interação familiar/acompanhante e equipe de enfermagem } \\
\text { no cuidado à criança hospitalizada: perspectivas para a a } \\
\text { enfermagem pediátrica }\end{array}$ & RJ/BR \\
\hline LILACS & 2010 & $\begin{array}{l}\text { Incertezas diante do Câncer infantil: compreendendo as } \\
\text { necessidades da mãe }\end{array}$ & SP/BR \\
\hline LILACS & 2008 & $\begin{array}{l}\text { A relação entre a equipe de enfermagem e o acompanhante } \\
\text { da criança hospitalizada: facilidades e dificuldades }\end{array}$ & SP/BR \\
\hline LILACS & 2006 & $\begin{array}{l}\text { A experiência de internação da família que vivencia a } \\
\text { doença e hospitalização da criança }\end{array}$ & SP/BR \\
\hline LILACS & 2006 & Sofrimento psíquico da família de crianças hospitalizadas & PR/BR \\
\hline MEDLINE & 2005 & $\begin{array}{l}\text { As informações recebidas pelos pais durante a hospitali- } \\
\text { zação do filho }\end{array}$ & SP/BR \\
\hline LILACS & 2005 & $\begin{array}{l}\text { Procurando manter o equilíbrio para atender suas demandas } \\
\text { e cuidar da criança hospitalizada: a experiência da família }\end{array}$ & SP/BR \\
\hline LILACS & 2004 & $\begin{array}{l}\text { Criança Hospitalizada: mãe e enfermagem compartilhan- } \\
\text { do o cuidado }\end{array}$ & PR/BR \\
\hline LILACS & 2002 & $\begin{array}{l}\text { Fatores que não apoiam a permanência de mães acompa- } \\
\text { nhantes em unidade de pediatria hospitalar }\end{array}$ & SP/BR \\
\hline LILACS & 2000 & $\begin{array}{l}\text { Vivenciando com o filho uma passagem difícil e reveladora } \\
\text { - a experiência da mãe acompanhante }\end{array}$ & SP/BR \\
\hline LILACS & 2000 & $\begin{array}{l}\text { A recepção da família na hospitalização de crianças } \\
\text { grupo de enfermeiras e cuidadores: convergências e di- } \\
\text { vergências em seus discursos }\end{array}$ & SP/BR \\
\hline LILACS & 1999 & SR \\
\hline
\end{tabular}




\section{DISCUSSÃO}

Em relação ao ano de publicação, observa-se um interesse crescente pela temática, com o aumento progressivo do número de estudos realizados, com destaque para o ano de 2010 com cinco produções, apesar do ECA ter sido promulgado em 1990. Vale destacar, que dos artigos selecionados, de acordo com os critérios de inclusão estabelecidos, o primeiro artigo publicado sobre a temática "Família da criança hospitalizada", tendo como sujeito o familiar/acompanhante na UIP/ACP foi em 1999, desenvolvido pela enfermagem de São Paulo, ou seja, nove anos após a promulgação do ECA.

No que se refere ao país de origem, constata-se que os estudos foram realizados no Brasil e, de acordo com a distribuição geográfica, verifica-se que o maior quantitativo de produção científica sobre a temática "família da criança hospitalizada" se concentra na região Sudeste com 10(66,7\%) artigos, seguida da região Sul com $5(33,3 \%)$ estudos; as regiões nordeste, centro-oeste e região norte não apresentaram nenhuma publicação científica sobre a temática na presente busca utilizando os critérios de inclusão e exclusão especificados. Esses dados evidenciam que nas regiões Sudeste e Sul do Brasil há um maior quantitativo destas publicações, levando a supor que esta tendência em parte esteja relacionada há maior concentração de programas de pós-graduação stricto senso, assim como de periódicos, vinculados a estes programas nas respectivas regiões.

Em relação à abordagem metodológica empregada, constata-se que há um predomínio dos estudos qualitativos com 13(86,7\%) artigos e apenas 3(13,3\%) estudos de natureza quantitativa. Por se tratar de estudos sobre a temática "familiar/acompanhante da criança hospitalizada" a utilização da abordagem metodológica qualitativa é bastante adequada para estudar experiência/vivências.

Vale destacar que entre os 13 artigos com abordagem qualitativa, apenas seis estudos explicitaram a utilização de referencial teórico para embasar a análise e interpretação do fenômeno estudado. Ou seja, cinco artigos utilizaram como referencial teórico o Interacionismo Simbólico de Charon e um utilizou o conceito de cultura de Geertz, abordagem centrada na criança e sua família de Elsen e Patrício e família saudável de Elsen. Quanto ao referencial metodológico, quatro utilizaram a Teoria Fundamentada nos Dados e um artigo utilizou o Interacionismo Interpretativo.

Foram identificadas 31 palavras-chave, sendo as mais utilizadas "criança hospitalizada" em 9(60\%) artigos, seguida de "família" em 8(53,3\%) artigos.
Percebe-se que apenas 3(20\%) artigos utilizaram a palavra-chave "mãe' e um $(6,6 \%)$ utilizou "pais". A totalidade dos estudos foi produzida pela enfermagem, apesar de não ter sido utilizado o descritor "enfermagem" ou "enfermagem pediátrica" para a realização da busca nas bases de dados. Dos artigos selecionados $10(66,6 \%)$ continham como descritor "enfermagem".

Verificou-se certa homogeneidade quanto à inclusão dos familiares que estavam acompanhando a criança durante a hospitalização, compondo amostras constituídas, majoritariamente, por mães. No que se refere à temática, após a leitura dos 15 artigos verificou-se a preocupação dos enfermeiros no desenvolvimento de pesquisas cujo participante seja a família da criança hospitalizada em relação aos seguintes temas: Interação entre a equipe de enfermagem e o acompanhante da criança hospitalizada, totalizando 6(40\%) artigos; Necessidades da família durante a hospitalização da criança, com 5(33,3\%) artigos; e Vivência/sentimentos da família da criança hospitalizada, 4(26,7\%) artigos. A síntese destes estudos será abordada a seguir.

Dos artigos selecionados, 6(40\%) têm como temática a interação da família com a equipe de enfermagem. Dentre as principais dificuldades identificadas na interação da família com a equipe de enfermagem está a falta de comunicação e de informação sobre o tratamento, além de tratamento desigual entre as crianças. Quanto as facilidades mais citadas nesta interação estão o fato do profissional ser bom tecnicamente, orientar sobre o tratamento, ter bom relacionamento, manter o diálogo, fazer a medicação na hora certa e prestar uma assistência adequada a criança ${ }^{(11)}$.

Corroborando, outro estudo afirma que quando a comunicação entre enfermeira e pais é eficiente, reduz-se a ansiedade dos pais e aumenta-se a aceitação desses na situação da doença e de hospitalização da criança, facilitando o regime de tratamento e favorecendo o processo de enfrentamento da doença, contribuindo para o crescimento enquanto indivíduo ${ }^{(12)}$.

Constata-se também em outro estudo que não há consenso, para a equipe de enfermagem, sobre a importância do acompanhante para a criança, nem tampouco os objetivos de sua permanência ao lado do filho, sem contar com o desconhecimento do papel que os familiares podem exercer, uma vez presentes. Sendo assim, as informações que os pais referiram receber durante a internação diziam respeito às rotinas, normas e regras do hospital, o que denota ainda que são as que mais preocupam a equipe, no sentido de que os pais não burlem estas normas para não interferir no trabalho que é realizado ${ }^{(13)}$. 
Enfermeiras e cuidadores apesar de pertencerem a universos distintos compartilham de pensamentos e percepções básicas e fundamentais a respeito da função do cuidador junto à criança hospitalizada, ficando demonstrado ainda que discordam em pontos, que em nosso entendimento podem ser contornados por meio da maior interação cuidador/família e enfermeira com a participação efetiva do cuidador/família na assistência da criança ${ }^{(14)}$.

Outro estudo identificou que os cuidados prestados pelo familiar/acompanhante no hospital são iguais aos desenvolvidos no domicilio, ficando sob a responsabilidade da equipe de enfermagem a prestação de cuidados de maior complexidade. Os familiares/ acompanhantes consideram a equipe de enfermagem atenciosa com seus filhos. Na admissão da criança podem ocorrer interferências como o medo e falta de confiança. Em contrapartida, conclui-se que os familiares/acompanhantes que permanecem por mais tempo na instituição ou que reinternam apreendem a cultura hospitalar utilizando a terminologia científica e elaborando estratégias para proteger a criança ${ }^{(4)}$.

Um dos artigos selecionados refere que a inclusão da mãe no cuidado ao filho hospitalizado tem acontecido de forma sutil, com essa assumindo procedimentos que são de competência da enfermagem e concentrando informação sobre o processo terapêutico. A falta de um projeto terapêutico coletivo sob a perspectiva do cuidado centrado na família foi identificado como sendo um dos óbices no envolvimento da mãe na assistência. Os resultados demonstram que as relações entre mãe e equipe de enfermagem mostram-se complexas e permeadas pelo exercício do poder, sendo necessária a busca da construção de um cuidado compartilhado ${ }^{(2)}$.

Quanto às necessidades do familiar que acompanha a criança hospitalizada, 5(33,3\%) estudos abordam a temática. A mãe apresenta a necessidade de estar presente acompanhando o tratamento da criança, ser amparada no momento de fraqueza, além da necessidade de manter o vínculo com a família. Suas necessidades, durante a internação da criança, tem caráter multidimensional, e seu reconhecimento é essencial para garantir a criação de um contexto de cuidado que potencialize o papel da mãe no suporte a criança ${ }^{(15)}$. Outro estudo detectou que uma das necessidades dos familiares é o interesse da equipe de saúde pelo paciente, e acredita-se que a experiência da família é facilitada quando é demonstrado interesse pela criança e sua família ${ }^{(16)}$.

Os pais têm necessidade de se sentirem acolhidos e seguros no ambiente hospitalar, sendo valorizadas ações do profissional de estar com a família, com- partilhando tanto a experiência da família quando o cuidado da criança. Promover um contexto relacional acolhedor, que permita à família sentir-se segura e que proporcione o desenvolvimento de novas competências e o encontro de recursos para o enfrentamento também é elemento fundamental do cuidado centrado na família, pois a tentativa de encontrar segurança em um ambiente não familiar é a principal necessidade dos familiares que vivem a experiência a hospitalização da criança e envolve tanto a segurança do familiar quanto a garantia de segurança da criança ${ }^{(17)}$.

Outro estudo aponta presença de outros familiares, a possibilidade de alimentar-se gratuitamente no hospital, a confiança na equipe hospitalar e a atenção que lhe é dada por esta equipe. Os fatores que não apoiam esta mãe são: a doença do filho, a preocupação com outras responsabilidades (exemplo: outros filhos, afazeres domésticos), a impossibilidade de sono e repouso, a falta de atenção da equipe e a impossibilidade de receber visitas ${ }^{(7)}$.

Outra necessidade da família é a construção de uma rede social na qual recebe apoio. A família valoriza o apoio recebido de seus familiares, das outras famílias com quem convive, dos amigos, vizinhos e profissionais de saúde; se fortalece na esperança e na fé em Deus e constrói vínculos. Conclui-se que conhecer as formas de apoio vivenciadas pelo familiar cuidador pode possibilitar a instrumentalização dos profissionais para um melhor direcionamento de ações e cuidados destinados à família e à criança hospitalizada ${ }^{(18)}$.

A temática sentimentos e vivências do familiar que acompanha a criança durante a hospitalização foi abordado por 4(26,7\%) estudos. Para a mãe, ela deve estar ao lado do filho passando tudo junto com ele durante a hospitalização. Logo, esta mãe se coloca na linha de frente, buscando manter suas emoções sob controle, fazendo tudo para defender a criança de mais sofrimento. $\mathrm{O}$ embate com o sofrimento possibilita à mãe pensar e enxergar a vida de outra maneira, uma vez que ela não vivencia o sofrimento pelo sofrimento, e esta situação a faz olhar para dentro de si, refletir e re-orientar as suas ações na vida ${ }^{(19)}$. A família espera proteger a criança da doença, da dor, do sofrimento emocional, dos procedimentos invasivos e da hospitalização ${ }^{(20)}$.

A mãe está exposta a pressões geradoras de sofrimento psíquico, expressando em atitudes agressivas, sentimentos de culpa, preocupações, medo; o sofrimento é desencadeado tanto pela estrutura física quanto pela organização do trabalho. As estratégias defensivas são evitar falar da hospitalização, chorar, desabafar, afastar-se do local gerador do sofrimento ${ }^{(21)}$. 
Ocorre também uma conduta passiva e dócil dos familiares frente às adversidades cotidianas, reflexos das próprias condições de poder e autoridade dos objetivos institucionais tão comuns nos cenários que desenvolvem ações de saúde na realidade brasileira. A emancipação poderia ser alcançada por meio de estratégias educativas caracterizadas pela informação e divulgação dos direitos do usuário e posturas criticas e reivindicatórias quando confrontados ${ }^{(22)}$.

\section{CONSIDERAÇÕES FINAIS}

Ao analisar as publicações selecionadas para este estudo, constata-se que o tema familiar/acompanhante da criança hospitalizada é um tema bastante presente na comunidade científica brasileira e que tem despertado principalmenteo interesse da Enfermagem.

Os estudos com famílias no cenário hospitalar pediátrico vêm sendo produzidos, progressivamente, a partir de 1999 e apontam para questões relacionadas à presença/permanência/participação da família no ambiente hospitalar. Os resultados demonstram a necessidade de maior sensibilização dos profissionais de saúde que veem este familiar apenas como um informante da criança. Ainda no aspecto da inserção das famílias no espaço intra-hospitalar, constata-se que se faz premente políticas públicas que amparem o familiar no contexto hospitalar pediátrico, principalmente no que se refere a infraestrutura para recebê-la.

A análise qualitativa dos estudos selecionados evidenciou, contudo, uma limitação quanto à seleção dos sujeitos, pois apesar de se proporem a estudar a família durante a hospitalização, a maioria dos estudos deu voz a apenas um familiar, geralmente aquele que se encontrava acompanhando a criança durante a hospitalização, majoritariamente mães, sem a inclusão de outros membros da unidade familiar.

Conclui-se que os estudos versam sobre questões ligadas de um modo geral a busca incessante dos autores em buscar compreender a subjetividade familiar; em contrapartida, a prática não reflete uma mudança de comportamento da equipe de saúde no que se refere à inclusão da família como parte do cuidado.

\section{REFERÊNCIAS}

1. Brasil. Ministério da Ação Social. Estatuto da criança e do adolescente. Lei n. 8.069, de 13 de junho de 1990. Dispõe sobre o Estatuto da Criança e do Adolescente, e dá outras providências. Diário Oficial da União, Brasília, DF, 1990 Jul 16. Seção 1: 13563.
2. Collet N, Rocha SMM. Criança hospitalizada: mãe e enfermagem compartilhando o cuidado. Rev. LatinoAm. Enfermagem. 2004;12(2):191-7.

3. Hockenberry MJ, Wilson D.Wong fundamentos de enfermagem pediátrica. Rio de Janeiro: Elsevier; 2011.

4. Souza TV, Oliveira ICS. Interação familiar/ acompanhante e equipe de enfermagem no cuidado a criança hospitalizada: perspectivas para a enfermagem pediátrica. Esc. Anna Nery. 2010;14(3):551-9.

5. Andraus LMS, Munari DB, Faria RM, Souza ACS. Incidentes críticos segundo os familiares de crianças hospitalizadas. Rev. enferm. UERJ. 2007;15(4):574-9.

6. Fernandes CNS, Andraus LMS, Munari DB. O aprendizado do cuidar da família da criança hospitalizada por meio de atividades grupais [Internet]. Rev. Eletr. Enf. 2006;8(1) [acesso em $10 \mathrm{dez}$ 2012]. Disponível: http:// www.fen.ufg.br/revista/revista8_1/original_14htm.

7. Siqueira LS, Sigaud CHS, Rezende MA. Fatores que apoiam e não apoiam permanência de mães acompanhantes em unidade de pediatria hospitalar. Rev Esc Enferm USP. 2002;36(3): 270-5.

8. Mariano ARL, Backes SD, Ilha S, Nicola ODG, Freitas BMH, Ferreira LLC. Significado da internação hospitalar pediátrica na perspectiva de profissionais e familiares. Cogitare enferm. 2011;16(3):511-6.

9. Ministério da Saúde(BR). Resolução da Diretoria Colegiada. RDC n. 50, de 21 de Fevereiro de 2002. Dispõe sobre o Regulamento Técnico para planejamento, programação, elaboração e avaliação de projetos físicos de estabelecimentos assistenciais de saúde. Diário Oficial da União, Brasília, DF, Diário Oficial da União - Seção 1 - 16/7/1990, Página 13563.

10. Mendes KDS, Silveira RCCP, Galvão. Revisão integrativa: método de pesquisa para a incorporação de evidências na saúde e na enfermagem. Texto Contexto Enferm. 2008;17(4): 758-64.

11. Soares MF, Leventhal LC. A relação entre a equipe de enfermagem e o acompanhante de criança hospitalizada: facilidades e dificuldades. Ciênc. cuid. saude. 2008;7(3):327-32.

12. Sabatés AL, Borba RIH. As informações recebidas pelos pais durante a hospitalização do filho. Rev. LatinoAm. Enfermagem. 2005;13(6):968-73.

13. Crepaldi MA, Varella PB. A recepção da família na hospitalização de crianças. Paidéia. 2000;10(19):33-9.

Cogitare Enferm. 2013 Out/Dez; 18(4):789-95 
14. Pai MM, Soares MAL. Percepção do significado da função do cuidador por um grupo do enfermeiras e cuidadores: convergências e divergências. Rev Esc Enferm USP. 1999;33(3): 231-5.

15. Angelo M, Moreira PL, Rodrigues, LMA. Incertezas diante do câncer infantil: compreendendo as necessidades da mãe. Esc. Anna Nery. 2010;14(2):301-8.

16. Pinto JP, Ribeiro RC, Silva CV. Procurando manter o equilíbrio para atender suas demandas e cuidar da criança hospitalizada: a experiência da família. Rev. Latino-Am. Enfermagem. 2005;13(6):974-81.

17. Silveira AO, Angelo M. Interaction experience for families who lives with their child's disease and hospitalization. Rev. Latino-Am. Enfermagem. 2006;14(6):893-900.

18. Gomes CG, Pintanel AC, Strasburg AC, Erdmann AL. O apoio social ao familiar cuidador durante a internação hospitalar da criança. Rev. enferm. UERJ. 2011;19(1):64-9.

19. Oliveira I, Angelo M. Vivenciando com o filho uma passagem difícil e reveladora: A experiência da mãe acompanhante. Rev Esc Enferm USP. 2000;34(2):2002-8.

20. Schultz LF, Llonch SA. Family living through sickness and child's hospitalization: qualitative study. Braz J Nurs. 2010;9(2).

21. Milanesi K, Collet N, Oliveira BRG. Sofrimento psíquico da família de crianças hospitalizadas. Rev. bras. enferm. 2006;59(6):769-74.

22. Werner W, Pedro ENR. Os múltiplos papéis sociais de mulheres cuidadoras-leigas de crianças hospitalizadas. Rev. Gaucha Enferm. 2010;31(2):335-42. 